\title{
On the growth of the Betti sequence of the canonical module
}

\author{
David A. Jorgensen - Graham J. Leuschke
}

Published online: 24 June 2008

C) Springer-Verlag 2008

\section{Erratum to: Math. Z. (2007) 256(3):647-659 DOI 10.1007/s00209-006-0096-x}

In our paper [1], three results appearing in Sect. 2 are incorrect as stated. The second part of Lemma 2.1, which assumes that $\operatorname{Ext}_{R}^{i}\left(M, N^{\vee}\right)=0$ for all $i$ in a certain range and concludes an inequality on the Betti numbers of $N$, is not true for the stated range of Ext-vanishing. The correction forces changes in the statements of Theorems 2.2 and 2.4 as well.

To correct the Lemma, we adjust the statement by moving the range of indices for which $\operatorname{Ext}_{R}\left(M, N^{\vee}\right)$ is assumed to vanish, adding the codimension of $M$ to both ends of the range. This preserves the statement for maximal Cohen-Macaulay modules, where our proof did in fact apply, and makes it true for Cohen-Macaulay modules of positive codimension. (We also reword the hypotheses slightly to try to avoid possible confusion).

Lemma 2.1 (corrected) Let $R$ be a CM local ring, $M$ a CM R-module of dimension d, and $N$ a MCM R-module. Let $n$ be an integer and assume that either

(1) $n \geq d+1$ and $\operatorname{Tor}_{i}^{R}(M, N)=0$ for all $i$ with $n-d \leq i \leq n$,or

(2) $n \geq 1$ and $\operatorname{Ext}_{R}^{i}\left(M, N^{\vee}\right)=0$ for all $i$ with $n+\operatorname{dim} R-d \leq i \leq n+\operatorname{dim} R$.

Then for any sequence $\boldsymbol{x}=x_{1}, \ldots, x_{d}$ regular on both $M$ and $R$,

$$
b_{n}(N) \leq \frac{\lambda(\mathfrak{m} M / x M)}{\mu(M)} b_{n-1}(N) .
$$

Moreover, equality holds if and only if both $\mathfrak{m}\left(M / x M \otimes_{R} N\right)=0$ and $\mathfrak{m}(\mathfrak{m} M / x M)=0$.

The online version of the original article can be found under doi:10.1007/s00209-006-0096-x.

D. A. Jorgensen

Department of Mathematics, University of Texas at Arlington, Arlington, TX 76019, USA

e-mail: djorgens@uta.edu

G. J. Leuschke $(\bowtie)$

Department of Mathematics, Syracuse University, Syracuse, NY 13244-1150, USA

e-mail: gjleusch@math.syr.edu 
The only change in the statement is in case (2), which in the original reads "for all $i$ with $1 \leq n \leq i \leq n+d$ ". Notice that if one were to add the additional assumption that $M$ were maximal Cohen-Macaulay, i.e. that $d=\operatorname{dim} R$, the two statements would coincide. The proof we gave (reproduced below) is correct in this case.

Proof We prove only (2).

Assume first that $M$ is MCM, so that $d=\operatorname{dim} R$, and induct on $d$. When $d=0$, Matlis duality yields $\operatorname{Tor}_{n}^{R}(M, N) \cong \operatorname{Ext}_{R}^{n}\left(M, N^{\vee}\right)^{\vee}=0$, and we get the inequality by case (1). When $d>0$, let overlines indicate reduction modulo $x_{d}$ and consider the sequence $0 \longrightarrow N^{\vee} \stackrel{x_{d}}{\longrightarrow} N^{\vee} \longrightarrow \overline{N^{\vee}} \longrightarrow 0$, which is exact as $N^{\vee}$ is MCM. Using the fact that $\operatorname{Hom}_{\bar{R}}(\bar{N}, \bar{\omega}) \cong \operatorname{Hom}_{R}(N, \omega) \otimes \bar{R}$, and the long exact sequence of Ext, we find that $\operatorname{Ext}_{R}^{i}\left(M, \bar{N}^{\vee}\right)=0$ for $n \leq i \leq n+(d-1)$. Since $\operatorname{Ext}_{R}^{i}\left(M, \bar{N}^{\vee}\right) \cong \operatorname{Ext}_{\bar{R}}^{i}\left(\bar{M}, \bar{N}^{\vee}\right)$ for all $i$, and $\bar{N}^{\vee}$ is MCM of dimension $d-1$ over $\bar{R}$, the induction hypothesis gives

$$
b_{n}^{\bar{R}}(\bar{N}) \leq \frac{\lambda(\overline{\mathfrak{m}} \bar{M} / \overline{\boldsymbol{x}} \bar{M})}{\mu(\bar{M})} b_{n-1}^{\bar{R}}(\bar{N}),
$$

which is equivalent to the inequality claimed.

Now assume that $M$ has codimension $c=\operatorname{dim} R-d \geq 1$. Let $\boldsymbol{y}=y_{1}, \ldots, y_{c}$ be a maximal $R$ - and $N^{\vee}$-regular sequence in the annihilator of $M$, and now let overlines indicate reduction modulo $\boldsymbol{y}$. Then $\operatorname{Ext}_{R}^{i}\left(M, N^{\vee}\right) \cong \operatorname{Ext}_{\bar{R}}^{i-c}\left(M, \overline{N^{\vee}}\right)$ for all $i \geq c$, so we see that $\operatorname{Ext} \frac{i}{R}\left(M, \bar{N}^{\vee}\right)=0$ for $n \leq i \leq n+\operatorname{dim} R-c=n+\operatorname{dim} \bar{R}$. As $M$ is MCM over $\bar{R}$, the previous case applies to give the inequality over $\bar{R}$, which again is equivalent to the inequality over $R$.

The statement about equality is unaffected by these changes.

We must also shift the range of vanishing Exts assumed in Theorem 2.2. The original text had $1 \leq i \leq d+\mu(\omega)$ in condition (2), but the corrected Lemma 2.1 leads to the following statement.

Theorem 2.2 (corrected) Let $(R, \mathfrak{m})$ be a CM local ring with canonical module $\omega$, and $M$ be a CM R-module of dimensiond such that for some sequence $\boldsymbol{x}$ of length d regular on both $M$ and $R$,

(1) $\lambda(\mathfrak{m} M / x M)<\mu(M)$, and

(2) $\operatorname{Ext}_{R}^{i}(M, R)=0$ for $1+\operatorname{dim} R-d \leq i \leq \operatorname{dim} R+\mu(\omega)$,

then $R$ is Gorenstein. The same statement, except allowing equality in (1), holds if either $\mathfrak{m}\left((M / \boldsymbol{x} M) \otimes_{R} \omega\right) \neq 0$ or $\mathfrak{m}(\mathfrak{m} M / \boldsymbol{x} M) \neq 0$.

Similarly, the statement of Theorem 2.4 must be corrected, replacing " $\operatorname{Ext}_{R}^{i}(M, R)=0$ for $1 \leq i \leq d+1$ " in condition (2) by the following.

Theorem 2.4 (corrected) Let $R$ be a generically Gorenstein CM local ring with canonical module $\omega$, and $M$ be a CM R-module of dimension d such that for some sequence $\boldsymbol{x}$ of length $d$ regular on both $M$ and $R$,

(1) $\lambda(\mathfrak{m} M / x M)<\mu(M)$, and

(2) $\operatorname{Ext}_{R}^{i}(M, R)=0$ for $1+\operatorname{dim} R-d \leq i \leq \operatorname{dim} R+1$, 
then $R$ is Gorenstein. The same statement, except allowing equality in (1), holds if either $\mathfrak{m}\left((M / \boldsymbol{x} M) \otimes_{R} \omega\right) \neq 0$ or $\mathfrak{m}(\mathfrak{m} M / \boldsymbol{x} M) \neq 0$.

The derivation of both Theorems from the Lemma is as in the original.

\section{Reference}

1. Jorgensen, D.A., Leuschke, G.J.: On the growth of the Betti sequence of the canonical module. Math. Z. 256(3), 647-659 (2007). doi:10.1007/s00209-006-0096-X 